\title{
MOYENS D'AMÉLIORER LA PRODUCTION DES ÉTANGS
}

\author{
Par M. Franz KRAATZ \\ Poissonnier à Grossbenten (Allemagne).
}

Tout le monde reconnaîtra qu'en France l'exploitation des étangs a fait beaucoup de progrès depuis vingt ans, surtout quant à la qualité du cheptel. La production pourrait encore être accrue considérablement, si l'on se résout à améliorer les étangs. Je sais bien que quelques propriétaires font une pisciculture tout à fait modernc et intensive, mais ils sont en petit nombre. Cependant, il serait temps que leur exemple fût suivi, car, dans tous les autres pays, les progrès déjà réalisés se poursuivent et s'amplifient. La Hongrie, par exemple, n'avait pas une pisciculture remarquable il y a 30 ans ; or, elle a exporté, pendant la saison écoulée, en Allemagne seulement et sans compter les autres pays, 668.400 kilos de Carpes. Il faut généraliser en France les méthodes éprouvées ailleurs, car la surproduction, jointe à la diminution du pouvoir d'achat du public engendrée par la crise économique, exercera une influence déprimante sur le prix international du Poisson d'eau douce. La France a exporté en Allemagne, pendant la campagne 1929-1930, 594. roo kilos de Carpes. Elle est donc intéressée à la question. Les propriétaires doivent compenser par une meilleure technique, tendant à l'abaissement des frais de production, la baisse prévisible des prix de vente à l'exportation.

Reconnaître la grande valeur du faucardage fui déjà un progrès important ; il m'est agréable de le voir appliqué par certains propriétaires qui repoussèrent nettement, il y a vingt ans, mes suggestions à ce sujet. Plusieurs de ces messieurs possèdent aujourd'hui leur bateau faucardeur (I).

Une autre dépense serait tout autant, et plus encore productive : débarrasser le sol de l'étang de la vase qui empêche le développement de la nourriture naturelle du Poisson. Il ne suffit pas du tout d'exposer à l'air la couche superficielle du sol, il importe que l'asséchement soit effectif sur trente ou quarante centimètres de profondeur au moins. Pour y arriver, il faudra peut-être creuser proportionnellement la rigole d'écoulement assez loin en aval de l'étang. Si le terrain ne permet pas ce travail, mieux vaut laisser la bonde ouverte toute une année. Je crois pouvoir garantir que les deux pêches consécutives, très souvent même la première seule, compensent cette perte d'une année, s'il a fallu s'y résoudre, car c'est seulement après

(I) J'ai eu, en effet, avant la guerre, époque où mon domicile était en France, la régie de plus de 600 hectares d'étangs dans les départements de Meurthe-et-Moselle et de Meuse. 
cet assec que le sol sera en situation de profiter des amendements. Un étang dont une bonne partie, ou au moins les bords, sont labourés chaque année et qui reçoit les engrais néccssaires, pourra être peuplé davantage et nécessite une plus grande quantité d'alevins.

Si beaucoup de propriétaires se décidaient à élever leur empoissonnage, ils ne seraient plus forcés d'en acheter ; cette économie est essentielle pour abaisser les prix de revient.

Une exploitation complète doit posséder plusieurs catégories d'étangs et des dépôts-réservoirs :

I $^{\circ}$ Frayère. - On a abandonné le système des bassins petits et peu profonds, car les nuits fraiches abaissent trop vite la température de l'eau, ce qui amène souvent la perte de tout l'effectif. Pour éviter ce danger, on emploie, depuis quelques années, des bassins d'au moins I.ooo mètres cubes, d'une profondeur d'un bon mètre à la bonde et de 6o centimètres à l'entrée de l'eau, qui doit toujours être courante. Ces frayères ne doivent être remplies que durant le temps où elles servent à la pose. Tout le reste de l'année, elles resteront en assec, couvertes de bons gazons d'herbes douces. Dès qu’on voit les tout petits Poissons, généralement au bout de trois ou quatre jours, il faut les transférer dans l'étang ci-après.

$2^{\circ}$ Etang d'alevinage. - Il peut mesurer de un demi à 5 hectares ; profond de deux mètres à la bonde et d'un mètre ailleurs, il est pourvu, en son milieu, d'un fossé large et profond et alimenté en permanence par un courant d'eau. Les Poissons ne doivent être pêchés qu'au printemps suivant, soit yo à I m mois après la naissance.

$3^{\circ}$ Etang de croissance. - Les Carpes d'un été sont mises dans un étang beaucoup plus grand que le précédent ef $y$ restent une année entière.

$4^{\circ}$ Etang de pêchr. - Les Carpes de deux étés sont finalement transférées dans le plus grand étang de l'exploitation où elles restent jusqu'à l'automne ou même jusqu'au second automne, car la mode revient des Carpes pleines d'cufs ou de laitance. Ifin que le Poisson ne risque rien pendant les un ou deux hivers, tous ces étangs doivent avoir dans la fosse du milieu et devant la vanne une profondeur d'environ 2 mètres.

$5^{\circ}$ Dépôt-réservoir. - Après la pếche, on entrepose les Carpes dans des réservoirs rectangulaires longs de so mètres et larges de to mètres, constamment alimentés d'eau courante, que l'on fait tomber de haut afin d'avoir tout l'oxyçène indispensable. Le sol des dépôts doit ître de bonne terre ; jamais de ciment, pierre ou bois ; il convient de les laisser à sec pendant tout l'été.

On peut, cependant, y faire frayer quelques géniteurs, mais on risque les changements brusques de température, enmme je le dis plus haut. En tout cas, il faut les garder à ser aussitôt après cet emploi.

Il est absolument nécessaire de donner à tous ces étangs, sur la majeure partie de leur surface, une profondeur de a môtres. On évitera la pêche 
en automne des Carpes de un à deux étés ; car, malgré toutes les précautions, ces jeunes Poissons sont blessés ; quand on les capture au printemps, ils sont plus robustes, ayant déjà recommencé à chercher leur nourriture ; ils souffrent moins des manipulations; par conséquent, les pertes seront moindres qu'aux pêches d'automne.

Pour avoir les diverses catégories d'étangs, décrites ci-dessus, on peut, si le terrain s'y prête, diviser un grand étang par des chaussées et en faire deux et trois plus petits.

Tous ces étangs doivent être bien cultivés et amendés, car les Carpes ayant bien profité, pendant leurs premier et deuxième étés, du sol préparé garderont l'habitude de bien grossir dans les troisième et quatrième étés.

Jusqu'ici, l'on a pensé avoir assez fait en répandant après la pêche, sur le sol à sec de l'étang, 200 kilos par hectare de chaux récemment éteinte. Encore le nombre des propriétaires français opérant de la sorte est-il, malheureusement, bien faible. Cette méthode ne fait pas tout. Elle est excellente pour désinfecter et désacidifier le sol, ou encore si l'étang a tendance à rester envasé. Mais l'eau de remplissage ne produisant d'effet que sur la couche supérieure du fond, il peut arriver que, dès Juin, diminue l'une ou l'autre des substances nécessaires au développement complet de la microflore et, par conséquent, de la microfaune. Il devient alors nécessaire et urgent d'y suppléer par des apports, répétés quatre ou cinq fois pendant l'été, de chaux en poudre ; on la dissémine du haut d'une barque, en dehors du courant s'il s'en trouve, jusqu'à un total de roo kilos à l'hectare.

A chaque étang doit être affectéc une barque rustique à fond large, pour le service du chaulage et pour permettre aussi à l'éleveur d'observer ses Poissons le plus souvent possible.

Un propriétaire allemand a construit un appareil fort simple déversant automatiquement, jour et nuit, pendant plusieurs mois d'été, de la chaux, en très faible quantité, dans l'eau qui alimente l'étang.

Si l'on n'utilise la chaux vive qu'au printemps, peu avant ou tout de suite après le remplissage, on peut épandre aussi du superphosphate, mais ro jours plus tard. Certains disséminent dans l'eau, à l'bectare, 25 à 30 kilos d'acide phosphorique avec r5 kilos de potasse, ou, encore, des scories mélangéeg à ces deux engrais et à la chaux vive. Mais il faut attendre Io jours après cet amendement pour l'empoissonnage.

Quand on peut trouver, à la ville proche, du fumier d'abattoirs, bon marché, par wagon complet, il convient aux étangs pauvres. On en fait de nombreux tas tout le long des bords de l'étang, dans l'eau.

Toutes ces pratiques augmentent la production et payent sûrement les frais et le travail. 